\title{
Alteración de la variabilidad del ritmo cardíaco en pacientes con síndrome coronario agudo sin supradesnivel del segmento ST. Experiencia preliminar
}

Carolina Jeria', Rodrigo Hernández¹, Claus Benn²

1 Hospital Clínico FACH

2 Universidad de Chile, Facultad de Medicina, ICBM

\section{Resumen:}

Antecentes: La variabilidad del ritmo cardiaco (VRC) es un indicador de la modulación que ejerce el sistema nervioso autónomo, el que puede ser analizado en los dominio de tiempo y de frecuencia. La disminución de VRC posterior al infarto agudo al miocardio con SDST se ha demostrado que se asocia a un aumento de muerte cardiovascular y arritmias ventriculares.

Objetivo: evaluar si una disminución de la VRC se correlaciona con el TIMI Risk Score y los marcadores séricos de daño miocárdico (CK mb masa y troponina $\mathrm{T}$ ) de los pacientes que cursan con un SCA $\sin$ SDST.

Métodos: Se incorporaron pacientes con el diagnóstico de SCA sin SDST dentro de las primeras 24 horas de hospitalización, en ritmo sinusal. Se excluyeron a pacientes con infarto miocárdico previo, insuficiencia cardiaca (NYHA III o IV), comorbilidad severa y arritmias. Se consignaron las características basales de los pacientes, el puntaje TIMI, los valores de las CK mb masa y troponinas. La VRC se obtuvo mediante un registro del ritmo cardiaco con el monitor Polar S810 durante 5 minutos en decúbito dorsal y se realizó el procesamiento de los datos con el software HRV Analysis 1.1.

Resultados: Se reclutaron 14 pacientes. Se observó una disminución significativa de los parámetros de VRC en relación a los valores definidos normales, para el dominio de tiempo (RR 902 ms, SD 23.41 ms, RMSSD 23.28 ms, pNN50 6\%). El análisis del grupo de pacientes que presentaron marcadores séricos de daño miocárdico positivos, obtuvo una correlación significativa con los parámetros de VFC en el dominio de frecuencia (alta frecuencia: HF $22.7 \mathrm{~ms} 2$ p:0.008, baja frecuencia: LF 66.9 u.n. p:0.016). El grupo de pacientes con CK mb masa elevada y troponinas positivas, registró valores significativamente menores de alta frecuencia (HF) y promedios significativamente mayores de las variables de baja frecuencia (LF), en relación al grupo CK mb masa baja $\mathrm{y}$ troponina negativa. Esto refleja un predominio de la regulación que ejerce del sistema nervioso simpático sobre la VRC y una disminución de la regulación parasimpática en este grupo de pacientes.

Conclusión: La evaluación de la VRC en un registro de 5 minutos en los pacientes con SCA sin SDST sugiere una disminución de la actividad del SNA parasimpático.

\section{Correspondencia:}

Dra. Carolina Jeria

Hospital Clínico FACH

carolajeria@gmail.com 


\section{Alterations in heart rhythm variability in patients with non-ST segment elevation acute coronary syndromes: preliminary report}

Background: Heart rhythm variability (HRV) is an indicator of autonomous nervous system modulation, which may be analyzed in both time and rate domains. A decrease in HRV following ST segment elevation acute myocardial infarction has been shown to be associated to increased cardiovascular mortality and ventricular arrhythmias.

Aim: To evaluate whether a decreased HRV is correlated to myocardial damage markers (CK MB mass and Troponin $\mathrm{t}$ ) in patients with a non-ST segment elevation acute coronary syndrome (NSTE-ACS)

Methods: Patients with NSTE-ACS in sinus rhythm within 24 hours of admission were included. We excluded patients with prior MI, heart failure class III or IV, arrhythmias or severe co-morbidity. Clinical characteristics, $\mathrm{CK}$ MB mass and serum troponin levels were determined. HRV was measured in a 5 min recording using the Polar S810 monitor with the patient lying flat. The software HRV Analysis 1.1 was used to process HRV data.

\section{Introducción}

La variabilidad del ritmo cardíaco (VRC) es la desviación de la media que los latidos cardíacos presentan en un determinado período de tiempo ${ }^{1}$ y representa una fina sintonía de los mecanismos de control del sistema autonómico del ritmo cardíaco latido a latido. Los eferentes simpáticos y vagales en el nodo sinusal tiene una participación constante y son modulados de manera central (centros respiratorios y vasomotor) y periférica (oscilación de la presión arterial, movimientos respiratorios y sistema humoral) lo que determina un acortamiento o alargamiento del ritmo sinusal en cada latido.

En la evolución del infarto agudo del miocardio (IAM) y en la insuficiencia cardíaca, un factor determinante en la sobrevida parece ser la disfunción en el equilibrio simpático-parasimpático, con un aumento de actividad simpática y/o una disminución de actividad parasimpática. La medición de la VRC corresponde a una técnica no invasiva y de bajo costo que ha demostrado ser un indicador pronóstico en el IAM con SDST 2,3,45; asimismo la disminución de la VRC en los pacientes con angina inestable e IAM sin SDST se relaciona con el aumento de morbimortalidad cardiovascular a corto y mediano plazo ${ }^{6,7}$.

De acuerdo al consenso de la Sociedad Europea de Cardiología y la Sociedad Norteamericana de Electrofisiología (Task Force) existen fundamentalmente dos períodos
Results: 14 patients were studied. In comparison with normal reference values, HRV in the time domain was significantly decreased (RR $902 \pm 23.4 \mathrm{~ms}$; RMSSD 23.38 ms; pNN50 6\%). Patients with positive myocardial damage markers exhibited altered HRV parameters in the frequency domain (high frequency: HF 22.7 ms2 p:0.008, low frequency: LF 66.9 u.n. p:0.016). Patients with high CK MB mass and elevated troponin levels had significantly lower high frequency values and significantly increased means for low frequency variables, compared to patients with normal CK MB mass and troponin levels. These findings reflect an increased sympathetic drive on HRV along with a decreased parasympathetic regulation in these patients.

Conclusion: Changes in 5 min recordings of HRV suggest a decreased activity of the para sympathetic system in patients with NSTE-ACS

Key words: Heart rhythm variability, acute coronary syndrome.

validados de registro del ritmo cardíaco: en 5 minutos y en 24 horas $^{8}$. Existen numerosas formas de análisis de la VRC que pueden ser agrupadas de la siguiente forma:

\section{Análisis en el Dominio del Tiempo.}

Utiliza medidas estadísticas, como la desviación estándar y la media aritmética para la cuantificación de los intervalos RR adyacentes de una serie temporal, que en ritmo sinusal incluyen a todos los intervalos entre complejos QRS. Esta forma de tratamiento de los datos resulta la más sencilla, pero impide distinguir cuál es la influencia autonómica dominante. No se detallará la nomenclatura de estas variables, ya que excede el objeto del trabajo y se puede obtener del documento de concenso Task Force. ${ }^{8}$

\section{Análisis en Dominio de Frecuencia}

a) Métodos no lineales: Scattograma (Diagrama de Poincarè). Es una representación en dos dimensiones en la cuál cada intervalo RR es graficado en función del intervalo RR que lo precede.

b) Métodos espectrales. Permiten obtener información concreta de la modulación que ejerce el sistema nervioso autónomo sobre la VRC. Es representada en las siguientes bandas de frecuencia:

- ULF (ultra baja frecuencia) $<0,003 \quad \mathrm{~Hz}$

- VLF (muy baja frecuencia) $<0,04 \quad \mathrm{~Hz}$

- LF (baja frecuencia): $\quad 0,04-0,15 \mathrm{~Hz}$ 
- HF (alta frecuencia): $\quad 0,15-0,40 \mathrm{~Hz}$

- VHF (muy alta frecuencia) $\quad 0,40-1,00 \mathrm{~Hz}$

La interpretación fisiológica de las bandas de frecuencia se puede resumir como sigue:

ULF: Expresa el ritmo circadiano de la VRC en registros de larga duración. Durante un registro de 24 horas el $90 \%$ de la VRC podría estar condicionada por los espectros de ULF y VLF.

VLF: En la actualidad no se sabe con exactitud el mecanismo fisiológico que regula el componente VLF.

LF: Refleja la estimulación o tono simpático, con modulación del parasimpático y en menor medida por el sistema renina-angiotensina, vasopresina y óxido nítrico.

HF: refleja la modulación del sistema parasimpático (tono vagal) sobre la VRC' .

Banda de muy alta frecuencia (VHF): Aparece en los registros durante la realización de ejercicio físico intenso. $\mathrm{Su}$ representación fisiológica necesita ser demostrada.

Cociente LF/HF: Es un indicador fiable del balance autonómico en reposo. ${ }^{10,11}$ Valores sobre este rango normal indicarán un mayor predominio simpático y valores por debajo reflejarán un mayor predominio parasimpático.

En la figura 1 se muestran los valores normales de los índices de VRC de acuerdo a la Task Force ${ }^{8}$.

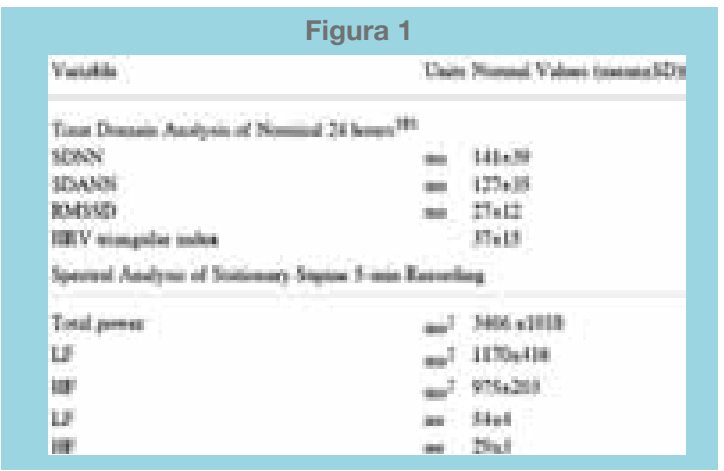

Valores normales de los parámetros de VRC. Reproducida de Task Force of the European Society of Cardiology the North American Society of Pacing Electrophysiology (1996).

Tradicionalmente la forma de obtener los intervalos RR para el análisis de variabilidad ha sido mediante la grabación de electrocardiograma (ECG) continuo de 3 o 12 derivaciones y la detección de los complejos QRS. Sin embargo, en las últimas décadas se ha desarrollado el monitoreo del ritmo cardíaco inalámbrico, entre los que destaca el dispositivo Polar S810®, que puede grabar intervalos RR para el posterior análisis de VRC12. Éste consta de un electrodo sujeto a una correa elástica que se posiciona en el tórax del paciente y detecta los intervalos RR con una resolución de $1 \mathrm{~ms}$; a su vez esta información es transmitida mediante radiofrecuencia al receptor que puede posicionarse en la muñeca del paciente. Posteriormente la información es transmitida mediante un cable a un computador con el software HRV Analysis 1.1, que procesa los datos y entrega la información de VRC del paciente estudiado. La validación de medición del dispositivo Polar S810 para la medición de los intervalos RR y el subsecuente análisis de la VRC en relación al "gold standard" del ECG, fue demostrada primero en ejercicio de baja intensidad ${ }^{12} y$ recientemente en reposo en posición supina ${ }^{13}$. No se han reportado estudios con grabaciones de corta duración del ritmo cardíaco con el monitor inalámbrico de VRC Polar S810 ® en pacientes con síndrome coronario agudo. El registro de corta duración del ritmo cardíaco en un equipo inalámbrico y el posterior análisis de la VRC, podría tener utilidad en la toma de decisiones clínicas en el paciente recientemente admitido en el hospital por un síndrome coronario agudo y además ser una herramienta complementaria para definir el pronóstico de este tipo de pacientes.

El objetivo del presente trabajo es evaluar si existe una correlación entre la VRC en un registro de cinco minutos, con el TIMI Risk Score y los marcadores séricos de daño miocárdico (CK mb masa y troponina $\mathrm{T}$ ) de los pacientes que cursan con un SCA sin SDST.

\section{Métodos}

Corresponde a un estudio descriptivo de una serie de casos.

\section{Pacientes}

Se incluyeron pacientes ingresados en el Hospital Clínico Gral. Dr. Raúl Yazigi desde diciembre 2007 a marzo del 2008, dentro de las primeras 24 horas de hospitalización, en ritmo sinusal y con el diagnóstico de ingreso de síndrome coronario agudo sin supradesnivel del segmento ST de acuerdo a los criterios establecidos por las guías de la AHA-ACC ${ }^{14}$ : dolor precordial característico, asociado o no a cambios electrocardiográficos (infradesnivel del segmento ST o inversión de la onda T). Se consideraron como criterios de exclusión el desarrollo de onda Q en el ECG, así como infarto agudo al miocardio ocurrido dentro de los 21 días previos, angioplastía coronaria dentro los 6 meses previos, insuficiencia cardíaca (NYHAFC III o IV), enfermedad severa crónica, presencia de fibrilación auricular, extrasístoles 
ventriculares o supraventriculares frecuentes, marcapaso uni o bicameral, desfibrilador o resincronizador, insuficiencia renal con creatinina de ingreso $>2.0 \mathrm{mg} / \mathrm{dL}$, shock, ventilación mecánica y dolor por cualquier causa en el momento de la medición. El protocolo de estudio fue aprobado por el comité de ética del hospital.

\section{Recolección de datos Clínicos}

Se consignaron las características basales y antecedentes médicos de los pacientes incluidos en el estudio, como los datos demográficos, clínicos, bioquímicos y diagnósticos previos. Se consideró como portadores de hipertensión arterial a quienes se les había diagnosticado, según el séptimo reporte del $\mathrm{JNC}^{15}$. Se definió diabetes de acuerdo a los criterios establecidos en el reporte The Expert Committe on the Diagnosis and Classification of Diabetes Mellitus ${ }^{16}$. Se consideró dislipidemia según los límites de colesterol, triglicéridos, HDL y LDL definidos por la ATP III ${ }^{17}$.

A cada paciente se le calculó el puntaje TIMI para síndrome coronario agudo sin elevación del segmento ST o angina inestable ${ }^{18}$.

\section{ECG, bioquímicos e imágenes}

Se analizaron la serie de ECG realizados a cada paciente en el servicio de urgencia y posteriormente cada 6 horas durante las primeras 24 horas de hospitalización. La depresión del segmento ST fue considera a 80 ms después del punto J. Tanto la inversión del segmento ST así como la inversión de las ondas T se consideraron significativas si ocurrían en dos derivaciones adyacentes y de por lo menos $0.1 \mathrm{mV}$.

Se realizaron tomas de muestras sanguíneas para el análisis de CK MB masa y troponina $\mathrm{T}$ de acuerdo al protocolo para los pacientes con dolor torácico del Hospital FACH que las considera al ingreso y cada seis horas hasta obtener el primer descenso en el valor de la CK MB masa. El valor superior de corte que utiliza el laboratorio para la $\mathrm{CK}$ MB masa es $4.9 \mathrm{pg} / \mathrm{dL}$ y para la troponina T es $0.01 \mathrm{pg} / \mathrm{dL}$. Para el análisis de los datos se consignaron los marcadores séricos de daño miocárdico que fueron tomados 6 horas posterior al ingreso del paciente al servicio de urgencias. La medición de CK MB masa y troponina T en el suero de los pacientes se realizó con un analizador Hitachi por el método de inmunoensayo Elicsys 2010. (Roche medical).

\section{Variabilidad del ritmo cardíaco}

Se obtuvo la grabación del ritmo cardíaco de cada pa- ciente mediante el monitor inalámbrico Polar S810®. Se registraron grabaciones de 5 minutos a cada paciente en posición de decúbito supino, en reposo, sin hablar, sin dolor, a una temperatura ambiente confortable (19 $21^{\circ} \mathrm{C}$ ). La información del monitor Polar fue transferida al Software Polar Precision Performance mediante un puerto USB infrarrojo y los datos de los intervalos RR fueron exportados en formato TXT para ser procesados en el software HRV Analysis 1.1.

Se realizó una selección de 300 latidos de los 5 minutos de grabación. Se obtuvieron los análisis en el dominio de tiempo y en el dominio de frecuencia que son presentados por el software, como se muestra en la figura 5 para cada uno de los pacientes incluidos en el estudio.

\section{Análisis estadístico}

Se calcularon los promedios de todos los parámetros de VRC con sus respectivas desviaciones estándar. Se realizó una correlación múltiple con el análisis de Spearman entre los parámetros de VRC (HF, LF, VLF, LF/HF, Pot Total, RMSSD, NN50, PNN50, SD1, SD2) y el puntaje TIMI, los valores de CK masa y troponina T. Se consideró un nivel de significancia de $\mathrm{p}<0,05$.

Se realizó una subdivisión de los pacientes en grupos pares entre las variables que evaluaron severidad del síndrome coronario agudo según mayor o menor gravedad como sigue: pacientes con troponina $\mathrm{T}$ positivas ( $>$ $0,011 \mathrm{pg} / \mathrm{dL})$ o negativas $(0,010 \mathrm{pg} / \mathrm{dL}) ; \mathrm{CK}$ masa alta $(>3,6 \mathrm{pg} / \mathrm{dL})$ o baja $(<3,6 \mathrm{pg} / \mathrm{dL})$; TIMI score mayor o igual a 3 o inferior a éste valor (Tabla 1 ). Se compararon los promedios de todos los parámetros de VRC de cada uno de estos pares grupos de pacientes, mediante prueba " $t$ " de Student para muestras independientes. Se consideró un nivel de significancia de $\mathrm{p}<0,05$.

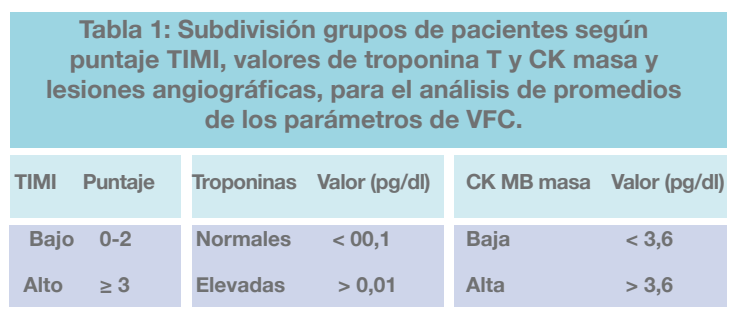

\section{Resultados}

Del total de 123 pacientes ingresados en la unidad coronaria del hospital FACH desde diciembre 2007 a marzo 2008, hubo 25 pacientes con diagnóstico de SCA sin SDST. De ellos se excluyeron 4 por presentar 
fibrilación auricular y 5 por enfermedad crónica severa o insuficiencia renal. En total 14 pacientes cumplieron con los criterios de inclusión y aceptaron formar parte del estudio. Las características basales se resumen en la Tabla 2.

\begin{tabular}{|c|c|}
\hline Variable & Pacientes \\
\hline Edad promedio - años (rango) & $65,4(48-88)$ \\
\hline Sexo $-n^{\circ}(\%)$ & 9 hombres $(64,2)$ \\
\hline HTA $-n^{\circ}(\%)$ & $12(85,7)$ \\
\hline Diabetes $-n^{\circ}(\%)$ & $3(21,4)$ \\
\hline Dislipidemia $-n^{\circ}(\%)$ & $9(64,2)$ \\
\hline Fumadores activos $-\mathrm{n}^{\circ}(\%)$ & $3(21,4)$ \\
\hline \multicolumn{2}{|l|}{ Historia de fármacos $-n^{\circ}(\%)$} \\
\hline -Aspirina & $10(71,4)$ \\
\hline -Betabloqueadores & $3(21,4)$ \\
\hline •IECA ○ ARAII & $11(78,6)$ \\
\hline Cardiopatía coronaria conocida* $-n^{\circ}(\%)$ & $4(28,5)$ \\
\hline Alteraciones $\mathrm{ECG}^{* \star}-\mathrm{n}^{\circ}(\%)$ & $5(35,7)$ \\
\hline TIMI - promedio (DS) & $3,2(1,62)$ \\
\hline Troponina T - promedio (DS) & $0,116(1,19)$ \\
\hline CK masa - promedio (DS) & $7,77(5,14)$ \\
\hline
\end{tabular}

Los valores presentados corresponden a los promedios de la variable indicada. Entre paréntesis se muestra el porcentaje (\%) de la variable en relación al total de pacientes o la desviación estándar (DS), según corresposponda. HTA: hipertensión arterial. IECA: inhibidor enzima convertidora de angiotensina. ARAII: antagonista de receptor de angiotensina. *: Antecedente de lesiones coronarias $>50 \%$ o prueba de isquemia positiva (cintigrama miocárdico de perfusión estrés-reposo o ecocardiografía de estrés **: infradesnivel del segmento $S T>$ o igual a $1 \mathrm{~mm}$ o inversión de la onda T que no tenía en ECG previos. ECG: electrocardiograma.

Se realizó el análisis de variabilidad del ritmo cardíaco, tanto en los dominios de tiempo como de frecuencia. Se obtuvo en la totalidad los pacientes una significativa disminución de todos los parámetros de variabilidad: promedios y desviaciones estándar en relación a los valores definidos normales. Los resultados se exponen en las tablas $3,4,5$ y 6 .

\begin{tabular}{|lccccc|}
\multicolumn{5}{c}{ Tabla 3: Promedio y desviaciones estándar de VRC en } \\
dominio de tiempo
\end{tabular}

FC: frecuencia cardíaca; Lpm: latidos por minuto; RR: intervalo $R R$; Entre paréntesis se muestran las desviaciones estándar.
Tabla 4: Promedio y desviaciones estándar de VRC en dominio de frecuencia.

\begin{tabular}{|c|c|c|c|}
\hline $\begin{array}{l}\text { Variable } \\
\text { Unidad }\end{array}$ & $\%$ (DS) & ms2 (DS) & u.n. (DS) \\
\hline VLF & $34,36(16,6)$ & $57,64(54,9)$ & \\
\hline LF & $34,9 \quad(14,2)$ & $77,42(107,3)$ & $55,82(21,15)$ \\
\hline HF & $30,73(17,7)$ & $70,14(73,3)$ & $44,17(21,15)$ \\
\hline LF/HF & $1,93(1,8)$ & & \\
\hline PT & & $202,35(224,3)$ & \\
\hline
\end{tabular}

Se muestran los promedios de las variables y entre paréntesis las desviaciones estándar (DS). VLF: banda de muy baja frecuencia, $L F$ : banda de baja frecuencia, $H F$ : banda de alta frecuencia, $L F /$ $H F$ : razón baja frecuencialalta frecuencia, PT: poder total.

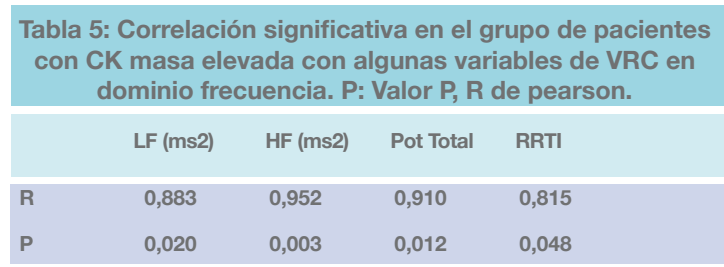

Tabla 6: Correlación significativa en el grupo de pacientes con Troponina positiva con algunas variables de VRC en dominio frecuencia. P: Valor P, R de pearson.

\begin{tabular}{cccccccc}
$\begin{array}{c}\text { VLF } \\
\text { (ms2) }\end{array}$ & $\begin{array}{c}\text { LF } \\
(\mathbf{m s} 2)\end{array}$ & $\begin{array}{c}\text { HF } \\
(\mathbf{m s} 2)\end{array}$ & $\begin{array}{c}\text { PT } \\
(\mathbf{m s} 2)\end{array}$ & $\begin{array}{c}\text { SD } \\
(\mathbf{m s})\end{array}$ & $\begin{array}{c}\text { RMSSD } \\
(\mathbf{m s})\end{array}$ & RRTI & $\begin{array}{c}\text { SD2 } \\
(\mathbf{m s})\end{array}$ \\
\hline R 0,871 & 0,774 & 0,876 & 0,902 & 0,828 & 0,722 & 0,886 & 0,744 \\
P 0,005 & 0,024 & 0,004 & 0,002 & 0,011 & 0,043 & 0,003 & 0,034
\end{tabular}

En el grupo del total de los pacientes incluidos en el presente estudio no se encontró una correlación significativa en el análisis de Spearman, entre los componentes de variabilidad del ritmo cardíaco y la severidad en la presentación del síndrome coronario agudo sin SDST, definida por score TIMI, CK MB masa y troponina T.

Al realizar la división según mayor o menor gravedad de presentación del SCA en los pacientes según TIMI, CK MB y Troponina, se obtuvo los subgrupos mostrados en la figura 2. Es así como fueron 9 pacientes que registraron TIMI > $3(64,3 \%)$ y 6 pacientes con CK MB masa elevada y troponina positiva $(35,7 \%)$.

Se efectuó el análisis de los promedios de los parámetros de VRC de los grupos troponina T positivas o negativas, CK masa alta o baja y TIMI score mayor o igual a 3 y menor a 3 respectivamente. Se obtuvo una diferencia significativa entre los promedios de HF, VLF, LF y LF/HF para los grupos de CK masa alta y baja. El grupo de pacientes con CK MB masa alta, muestra una disminución del promedio de $\mathrm{HF}$ (\%, ms2 y u.n.), y asimismo un aumento 


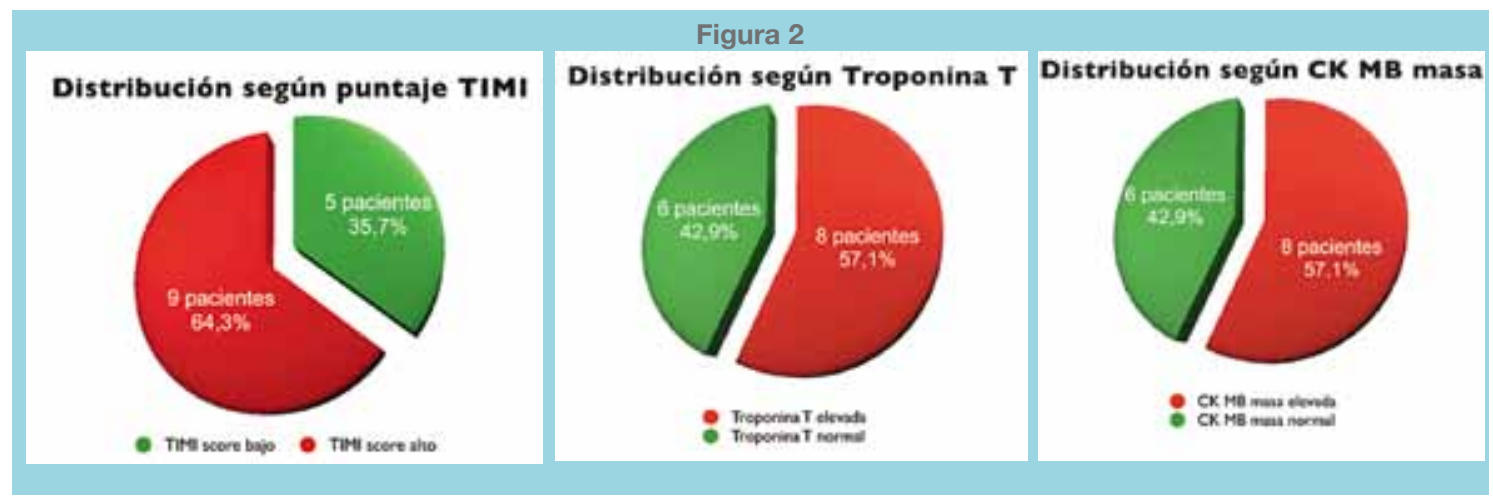

División del grupo de pacientes según gravedad de presentación del SCA según TIMI, CK MB masa y Troponina.

del promedio de LF (ms2). Estos resultados se muestran en las figuras 3 y 4 . En la figura 5 se muestra que el grupo de pacientes con troponina $\mathrm{T}$ positiva tuvo un promedio de HF ms2 significativamente más bajo que el grupo de pacientes con troponina $\mathrm{T}$ negativas.

Al realizar un análisis de correlación de Spearman entre los parámetros de variabilidad en la población de mayor riesgo, es decir los pacientes con troponina $\mathrm{T}$ positivas, CK masa elevada y TIMI > 3, se obtuvieron los resultados expresados en las tablas 5 y 6 que muestran los valores de R de Pearson y P. Se observa que existe una buena correlación en el grupo de troponina $\mathrm{T}$ positivas con las variables LF (ms2), HF (ms2), Potencia total, RRTI. Así mismo, en el grupo de CK masa elevada se muestra una buena correlación con VLF (ms2), LF (ms2), HF (ms2), Potencia total (ms2), SD (ms), RMSSD (ms), RRTI, SD2 (ms). No hubo correlación entre los pacientes con puntajes TIMI > 3 y los parámetros de VRC.
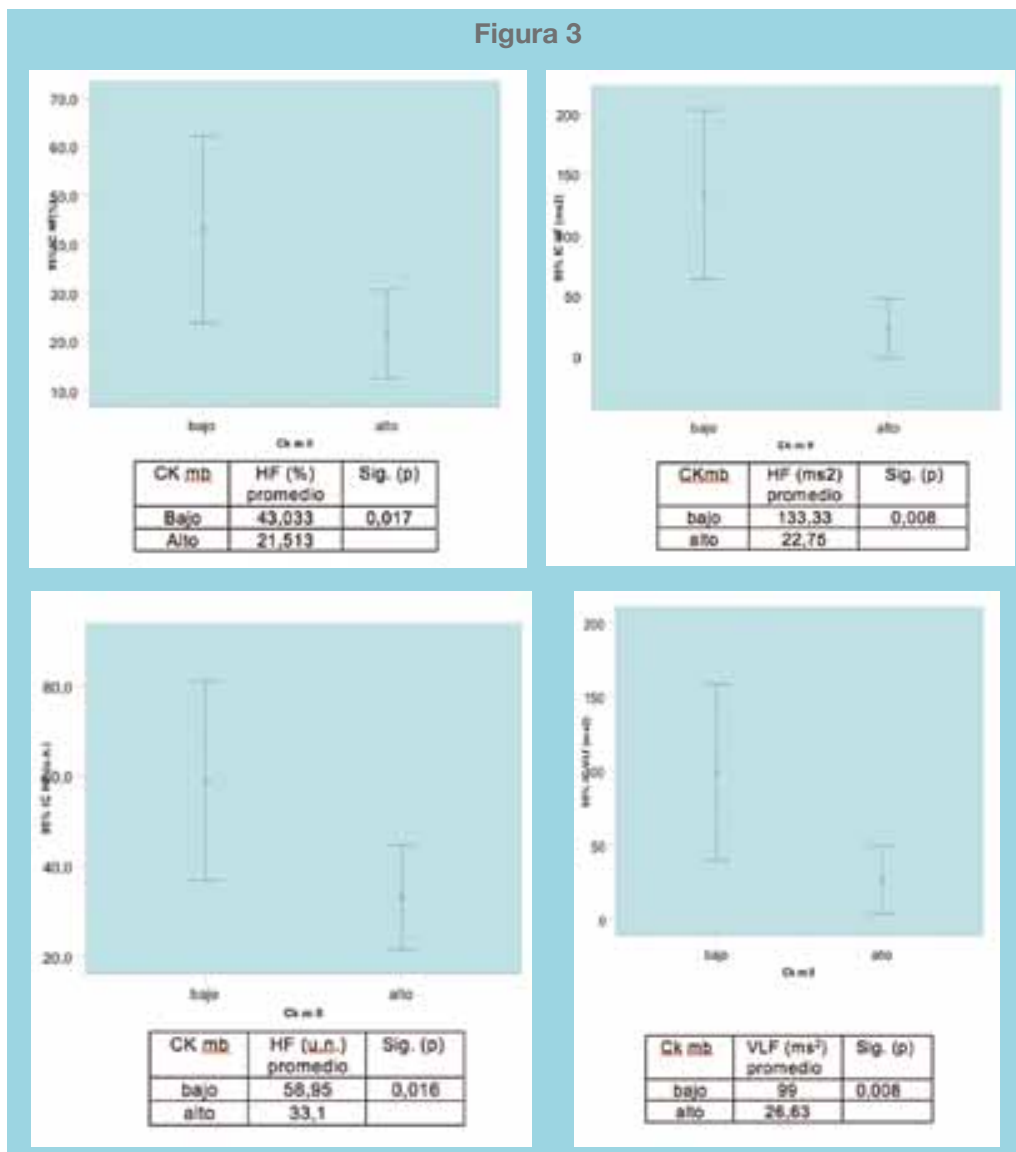

Gráficos con barras de error que compara los promedios de $\mathrm{HF}(\%, \mathrm{~ms} 2 \mathrm{y}$ u.n.) y VLF (ms2) para los grupos de CK masa alto y bajo. Sig: significancia, Ckm II: CK masa. 

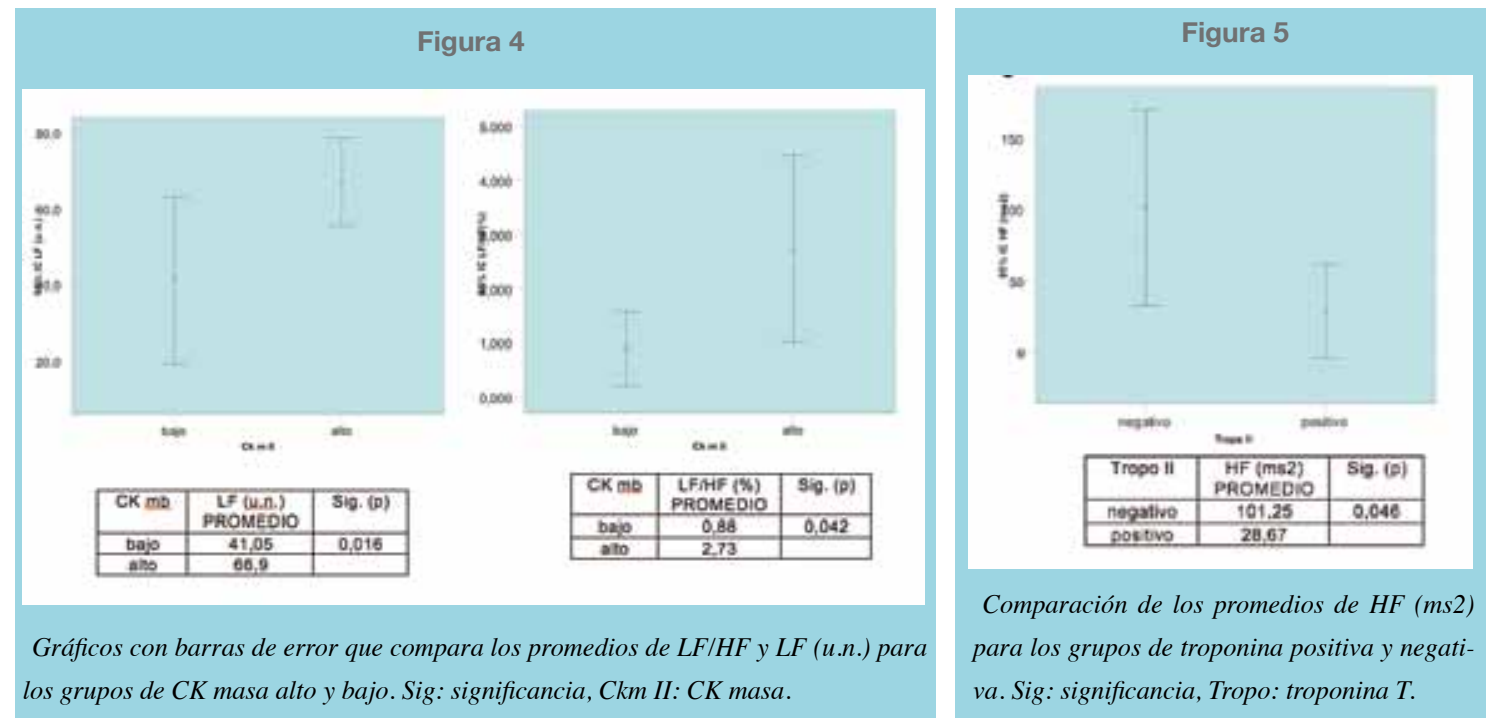

\section{Discusión}

En los pacientes con síndrome coronario agudo sin SDST incluidos en el presente estudio, se observó una disminución de los valores de los diferentes parámetros que definen la VRC. El grado de disminución no obtuvo una correlación estadísticamente significativa con la severidad del síndrome coronario, expresada por el puntaje TIMI, el valor de troponina $\mathrm{T}$ y $\mathrm{CK}$ masa. La no dependencia entre estos dos grupos de variables, se puede explicar en parte por el tamaño muestral. Con un $\mathrm{N}$ de 14 pacientes se pueden acentuar las diferencias que debieran existir en la VRC de los pacientes por edad, género y condiciones basales.

Cabe destacar que el número de pacientes diabéticos y usuarios de fármacos betabloqueadores incluidos en el estudio, es bajo y similar al porcentaje reportado en ensayos multicéntricos que han demostrado no interfierir con los resultados de morbimortalidad en pacientes con infarto agudo al miocardio o SCA. Sin embargo, debido al pequeño número de pacientes del presente estudio, éste puede ser un ítem a considerar en el análisis de VRC. Se ha demostrado que los pacientes diabéticos tienen una alteración de la regulación del sistema autonómico, con una leve disminución de los parámetros espectrales de la VRC que se ven considerablemente modificados en aquellos pacientes que presentan daño de órgano blanco, como en la neuropatía diabética ${ }^{22,23}$.

Otra debilidad del estudio hace referencia a no tener un grupo de control, ya que si bien es cierto existen valores poblaciones definidos como normales, puede ser de utilidad tener un rango de referencia para una muestra de pacientes de características basales similares. Sin embargo, esto no aseguraría la aleatoriedad de la muestra.

Al comparar los parámetros de VRC en los subgrupos de mayor y menor riesgo de TIMI $(>3,<3)$, troponina $\mathrm{T}$ (positivas y negativas) y CK masa (altas y bajas), se observó una interesante correlación. El grupo de troponina $\mathrm{T}$ positivas presentó el promedio de banda de alta frecuencia (HF ms2) más baja que el grupo de troponina $\mathrm{T}$ negativas (figura 5). Esto implica una baja actividad parasimpática, que es considerada protectora del sistema cardiovascular. Así mismo en el grupos de CK MB masa elevada se observó una disminución del promedio de la banda de alta frecuencia (HF en \%, ms2 y u.n.) y un aumento del promedio tanto de la banda de baja frecuencia (LF ms2), así como del índice LF/HF. Esto se interpreta como una disminución de la actividad parasimpática y un aumento del predominio de la actividad simpática en el grupo de pacientes con CK MB masa elevado (figuras 3 y 4). Estos hallazgos son concordantes con el comportamiento que debiera tener el sistema nervioso simpático en los síndromes coronarios agudos, con aumento de la actividad simpática y disminución parasimpática que ha sido descrita en la literatura. Existen series pequeñas de pacientes con síndrome coronario agudo sin supradesnivel del segmento ST en los que la disminución de VRC se correlaciona con el pronóstico y el valor predictivo de nuevos eventos cardiovasculares en el corto plazo y mediano plazo ${ }^{19,20,7}$. Así también se ha descrito que la VRC combinado con otros predictores clínicos, de laboratorio y ECG de mortalidad 
en IAM a 12 meses, tienen un excelente valor predictivo negativo ${ }^{21}$.

Recientemente se dio a conocer un trabajo que vincula la regulación de las señales adrenérgicas con la expresión de genes que controlan el metabolismo oxidativo en el músculo esquelético ${ }^{24}$. Esto demuestra la relación antes supuesta entre daño miocárdico y estrés oxidativo, lo que abre otra senda en la investigación relacionada a la VRC. Existen numerosas publicaciones acerca de VRC en las últimas décadas y entregan evidencia que ésta es una herramienta pronóstica en diferentes patologías cardiovasculares. Sin embargo, no es una medición que esté incorporada a la práctica clínica habitual. Esto probablemente se deba a que no existe consenso en la utilización de un único parámetro que refleje la VRC, sino que existen muchos índices que pueden llegar a ser confundentes para el médico. Además la disminución de la VRC en las diferentes patologías, es en muchos casos multifactorial lo que dificulta el diseño de una estrategia terapéutica específica. Un ensayo de evaluación de la VRC con intervención terapéutica es el subestudio del EMIAT (European Myocardial Infarct Amiodarone) ${ }^{25}$, que identifica a los pacientes con disminución de la VRC y muestra que se podrían beneficiar de amiodarona profiláctica post infarto agudo al miocardio, con disfunción ventricular sistólica moderada.

El registro corto de 5 minutos del ritmo cardíaco para el posterior análisis de variabilidad, está validado desde el consenso de la Sociedad Europea de Cardiología en 1996. Hay que mencionar que se limita la utilidad de los índices del dominio de tiempo, excepto RMSS (ms) y SDNN (ms), siempre que comparen registros de la misma duración en cantidad de latidos. Para grabaciones de 5 minutos se utiliza preferentemente los métodos espectrales: $\mathrm{HF}$, LF, VLF, LF/HF, poder total26. Steeds ${ }^{27}$ reportó la medición de VRC con holter de ECG durante 5 minutos en 164 pacientes a las 48 horas post infarto agudo. Dicho estudio demostró que la disminución de la VRC se asoció a un aumento de eventos adversos cardiovasculares.

El registro del ritmo cardíaco con el monitor Polar S810® se ha realizado en personas sanas bajo condiciones de entrenamiento cardiovascular y en sujetos en reposo en diferentes condiciones medioambientales. Como se mencionó previamente fue Francois Gamelin ${ }^{13}$ quién realizó la validación del equipo Polar para el análisis de VRC. No se han reportado estudios de VRC que contemplen mediciones con un monitor inalámbrico en pacientes con síndromes coronarios agudos. Es así como el presente estudio, se podría considerar una contribución en este ámbito, ya que debido al bajo costo, facilidad de registro y procesamiento de los datos, puede ser una herramienta útil en la práctica clínica, en lugares aislados o con bajos recursos. Se requieren más estudios que corroboren estos hallazgos.

\section{Conclusión:}

En los 14 pacientes que fueron parte del presente estudio con síndrome coronario agudo sin SDST sin falla ventricular, existe una relación entre la regulación del ritmo cardíaco por el sistema nervioso autónomo y la elevación de marcadores séricos de daño miocárdico.

Agradecimientos:

Kinesiólogo PhD Jorge Cancino, Dr. José Luis Vukasovic.

\section{Referencias:}

1. VUKASOVIC JL, FLORENZANO F. Variabilidad de la Frecuencia Cardiaca: sus fundamentos fisiopatológicos y su utilidad como índice pronóstico post-infarto agudo del miocardio. Rev Med Chile 1995;123:1412-1417

2. BIGGER JT JR, FLEISS JL, STEINMAN RC, ROLNITZKY LM, KLEIGER RE, ROTTMAN JN. Correlations among time and frequency domain measures of heart period variability two weeks after acute myocardial infarction. Am J Cardiol 1992; 69:891-8.

3. BIGGER JT JR., FLEISS JL, STEINMAN RC, ROL-
NITZKY LM, KLEIGER RE, ROTTMAN JN. Frequency domain measures of heart period variability and mortality after myocardial infarction. Circulation 1992; 85:164-71.

4. CASOLO GC, STRODER P, SIGNORINI C, CALZOLARI F, ZUCCHINI M, BALLI E, et al. Heart rate variability during the acute phase of myocardial infarction. Circulation 1992; 85:2073-9.

5. KLEIGER RE, MILLER JP, BIGGER JT JR., MOSS AJ Decreased heart rate variability and its association with increased mortality after acute myocardial infarction. Am J Cardiol 1987; 59:256-62.

6. LORICCHIO ML, DI CLEMENTE D, SACCONE V, 
VENTURI P, BORGHI A, BUGIARDINI R. Prognostic value of heart rate variability in unstable angina. $\mathrm{J}$ Am Coll Cardiol 1994; 21:197A.

7. LANZA GA, CIANFLONE D, REBUZZI AG, ANGELONI G, SESTITO A, CIRIELLO G, et al. Prognostic value of ventricular arrhythmias and heart rate variability in patients with unstable angina. Heart 2006; 92:1055-1063.

8. Task Force of the European Society of Cardiology the North American Society of Pacing Electrophysiology. Heart Rate Variability: Standards of Measurement, Physiological Interpretation, and Clinical Use. Circulation 1996; 93:1043-1065.

9. POMERANZ B, MACAULAY RJ, CAUDILL MA, KUTZ I, ADAM D, GORDON D, et al. Assessment of autonomic function in humans by heart rate spectral analysis. Am J Physiol 1985; 248:H151-3.

10. PAGANI, M. PAGANI M, MONTANO N, PORTA A, MALLIANI A, ABBOUD FM, BIRKETT C, et al. Relationship Between Spectral Components of Cardiovascular Variabilities and Direct Measures of Muscle Sympathetic Nerve Activity in Humans. Circulation 1997; 95: $1441-1448$.

11. JENSEN-URSTAD K, JENSEN-URSTAD K, STORCK N, BOUVIER F, ERICSON M, LINDBLAD LE, JENSEN-URSTAD M, et al. Heart rate variability in healthy subjects is related to age and gender. Acta Physiol Scand. 1997; 160: 235-241.

12. KINGSLEY M, LEWIS MJ, MARSON RE. Comparison of polar $810 \mathrm{~s}$ and an ambulatory ECG system for RR interval measurement during progressive exercise. Int. J. Sports Med. 2005; 26:39-44.

13. GAMELIN FX, BERTHOIN S, BOSQUET L. Validity of the polar $\mathrm{S} 810$ heart rate monitor to measure R-R intervals at rest. Med Sci Sports Exerc. 2006; 38: 887-93.

14. ACC/AHA 2007 guidelines for the management of patients with unstable angina/non ST-elevation myocardial infarction: a report of the American College of Cardiology/American Heart Association Task Force on Practice Guidelines. 2007;116: e148-304.

15. CHOBANIAN AV, BAKRIS GL, BLACK HR, CUSHMAN WC, GREEN LA, IZZO JL JR, et al. The Seventh Report of the Joint National Committee on Prevention, Detection, Evaluation, and Treatment of High Blood Pressure: The JNC 7 report. JAMA 2003; 289:2560-72.

16. Report of the Expert Committee on the Diagnosis and
Classification of Diabetes Mellitus. Diabetes Care 2003; 26: S5-S20.

17. Third Report of the National Cholesterol Education Program (NCEP) Expert Panel on Detection, Evaluation, and Treatment of High Blood Cholesterol in Adults (Adult Treatment Panel III) final report. Circulation 2002; 106: 3143-421.

18. ANTMAN E, COHEN M, BERNINK P, MCCABE C, HORACEK T, PAPUCHIS G, et al. The TIMI Risk Score for Unstable Angina/Non-ST Elevation MI. JAMA 2000; 284:835-842.

19. HUANG J, LEATHAM E, REDWOOD S, YI G, CHEN L, KASKI JC, et al. Heart rate variability is depressed in patients with unstable angina. J Am Coll Cardiol 1994; 23:196A.

20. HUANG J, SOPHER M, LEATHAM E, REDWOOD S, CAMM A, KASKI JC. Heart rate variability depression in patients with unstable angina. Am Heart 1995; 130: 772-9.

21. KENNON S, PRICE CP, MILLS PG, MACCALLUM PK, COOPER J, HOOPER J, et al. Cumulative risk assessment in unstable angina: clinical, electrocardiographic, autonomic, and biochemical markers. Heart 2003; 89:36-41.

22. PAGANI M, MALFATTO G, PIERINI S, CASATI R, MASU AM, POLI M, et al. Spectral analysis of heart rate variability in the assessment of autonomic diabetic neuropathy. J Auton Nerv Syst 1988; 23:143-53.

23. O'BRIEN IA, O'HARE JP, LEWIN IG, CORRALL RJ. The prevalence of autonomic neuropathy in insulin-dependent diabetes mellitus: a controlled study based on heart rate variability. Q J Med 1986; 61: 957-67.

24. PEAREN MA, MYERS SA, RAICHUR S, RYALL JG, LYNCH GS, MUSCAT G. The Orphan Nuclear Receptor, NOR-1, a Target of \{beta\}-Adrenergic Signaling, Regulates Gene Expression that Controls Oxidative Metabolism in Skeletal Muscle. Endocrinology 2008; 149, 2853-2865

25. MALIK M, CAMM AJ, JANSE MJ, JULIAN DG, FRANGIN GA, SCHWARTZ PJ. Depressed heart rate variability identifies postinfarction patients who might benefit from prophylactic treatment with amiodarone: a substudy of EMIAT (The European Myocardial Infarct Amiodarone Trial). J Am Coll Cardiol. 2000; 35:1263-75.

26. SINNREICH R, KARK JD, FRIEDLANDER Y, SAPOZNIKOV D, LURIA MH. Five minute recording of heart rate variability for population studies: repeatability and age-sex characteristics. Heart 1998; 80: 156-162. 